\title{
Application of the Method of Auxiliary Sources for the Analysis of Plane-Wave Scattering by Impedance Spheres
}

\section{Karamehmedovi, Mirza; Breinbjerg, Olav}

Published in:

Proceedings of International Conference on Mathematical Methods in Electromagnetic Theory

Publication date:

2002

Document Version

Publisher's PDF, also known as Version of record

Link back to DTU Orbit

Citation (APA):

Karamehmedovi, M., \& Breinbjerg, O. (2002). Application of the Method of Auxiliary Sources for the Analysis of Plane-Wave Scattering by Impedance Spheres. In Proceedings of International Conference on Mathematical Methods in Electromagnetic Theory (Vol. 2, pp. 499-501). IEEE.

\section{General rights}

Copyright and moral rights for the publications made accessible in the public portal are retained by the authors and/or other copyright owners and it is a condition of accessing publications that users recognise and abide by the legal requirements associated with these rights.

- Users may download and print one copy of any publication from the public portal for the purpose of private study or research.

- You may not further distribute the material or use it for any profit-making activity or commercial gain

- You may freely distribute the URL identifying the publication in the public portal 


\title{
APPLICATION OF THE METHOD OF AUXILIARY SOURCES FOR THE ANALYSIS OF PLANE-WAVE SCATTERING BY IMPEDANCE SPHERES
}

\author{
Mirza Karamehmedović, Olav Breinbjerg \\ $\emptyset$ rsted $\bullet$ DTU, Electromagnetic Systems, Technical University of Denmark \\ Ørsteds Plads, building 348, DK-2800 Kgs. Lyngby, Denmark \\ e-mail: mirza@ieee.org, ob@oersted.dtu.dk
}

\begin{abstract}
The Method of Auxiliary Sources (MAS) is applied to 3D scattering problems involving spherical impedance scatterers. The MAS results are compared with the reference spherical wave expansion (SWE) solution. It is demonstrated that good agreement is achieved between the MAS and SWE results.
\end{abstract}

\section{INTRODUCTION}

The Method of Auxiliary Sources (MAS) is a numerical technique applicable to electromagnetic scattering problems. In the general case, a set of spatially impulsive electric and/or magnetic sources is introduced to radiate an approximation to the unknown scattered field. These so-called auxiliary sources are located on an auxiliary surface, typically conformal to, and enclosed within, the physical surface of the scatterer. Point matching of the boundary condition on the physical surface is enforced to determine the complex amplitudes of the auxiliary sources. The MAS originates from an application of a special case of the Method of Moments (MoM), utilising spatially impulsive expansion and testing functions, to a generalised surface integral equation formulation [1]. An overview of MAS is given in [2]. Utilisation of MAS for numerical solution of various 2D scattering problems and 3D PEC and dielectric scattering problems has been reported earlier [3], [4], [5]. The purpose of this work is to investigate the performance of MAS when the method is applied to scattering problems involving spherical impedance scatterers. The spherical wave expansion (SWE) solution is developed and used as a reference. The scattering problem under investigation is

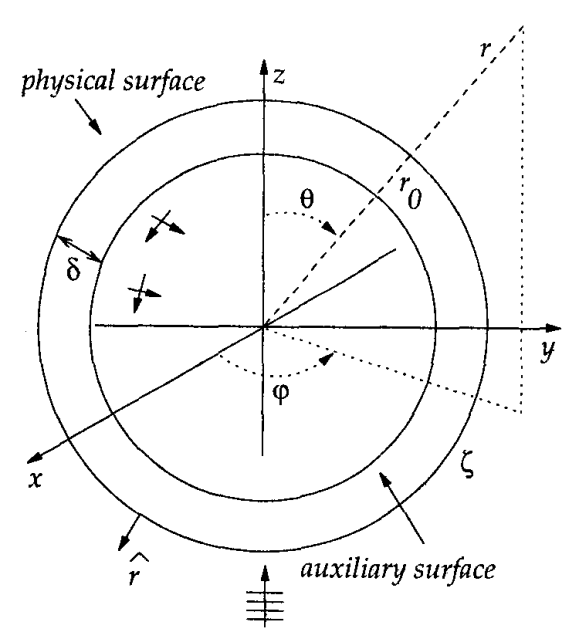
illustrated in Figure 1, together with the introduced Cartesian and spherical co-ordinate systems. The spherical scatterer of radius $r_{0}$ is illuminated by an $x$-polarised uniform plane wave $\left(\mathbf{E}^{i}, \mathbf{H}^{i}\right)$ of wavelength $\lambda$ and propagating in the $z-$ direction. The Standard Impedance Boundary Condition (SIBC) holds on the physical surface of the scatterer, $\hat{\mathbf{r}} \times(\mathbf{E} \times \hat{\mathbf{r}})=\zeta \hat{\mathbf{r}} \times \mathbf{H}$. The chosen auxiliary sources are pairs of crossed $\hat{\theta}$ - and $\hat{\varphi}$ directed electric Hertzian dipoles located on a sphere of radius $r_{0}-\delta$. The auxiliary source pairs, as well as the matching points, are placed equidistantly in the angular co-ordinates $(\theta, \varphi)$. The total number of source pairs is denoted by $N$.

Figure 1: The scattering problem geometry

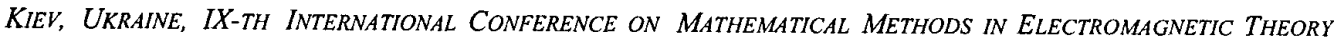


The SWE solution is given by (see [6, Chapter 2] for definitions of symbols)

$$
\begin{aligned}
& \mathbf{E}^{s}= k / \sqrt{\eta} \sum_{s, m, n} Q_{s, m, n}^{(3)} \mathbf{F}_{s, m, n}^{(3)}, \mathbf{H}^{s}=-i k \sqrt{\eta} \sum_{s, m, n} Q_{s, m, n}^{(3)} \mathbf{F}_{3-s, m, n}^{(3)}, Q_{s, m, n}^{(3)}=E^{i} a / b, \\
& a= i^{n+1} \sqrt{\pi(2 n+1)}(-1)^{4-s-m} R_{3-s, n}^{(3)} R_{s, n}^{(1)}\left(-\delta_{s, 1}\left(\delta_{m, 1}+\delta_{m,-1}\right)+\delta_{s, 2}\left(\delta_{m,-1}-\delta_{m, 1}\right)\right)- \\
& i^{n+2} \zeta \eta \sqrt{\pi(2 n+1)}\left(-\delta_{s, 1} R_{2, n}^{(1)} R_{2, n}^{(3)}\left(\delta_{m, 1}+\delta_{m,-1}\right)+\delta_{s, 2} R_{1, n}^{(1)} R_{1, n}^{(3)}\left(\delta_{m,-1}-\delta_{m, 1}\right)\right), \\
& b=-k / \sqrt{\eta}(-1)^{3-m-s} R_{3-s, n}^{(3)} R_{s, n}^{(3)}+\zeta i k \sqrt{\eta}(-1)^{m}\left(R_{3-s, n}^{(3)}\right)^{2}, \\
& R_{1, n}^{(3)} \equiv h_{n}^{(1)}\left(k r_{0}\right), R_{2, n}^{(1)} \equiv j_{n}^{\prime}\left(k r_{0}\right), R_{2, n}^{(3)} \equiv h_{n}^{(1)^{\prime}}\left(k r_{0}\right) .
\end{aligned}
$$

NUMERICAL RESULTS

We define the boundary condition error (BCE) by

$$
B C E \equiv \frac{1}{M N\left|\mathbf{E}^{i}\right|} \sum_{m, n}^{M, N}\left|\hat{\mathbf{r}}_{m, n} \times\left(\mathbf{E}_{m, n} \times \hat{\mathbf{r}}_{m, n}\right)-\zeta \hat{\mathbf{r}}_{m, n} \times \mathbf{H}_{m, n}\right|
$$

The summation is performed over the scatterer physical surface. Figure 2 shows the BCE as a function of $N$ for four different scatterer surface impedances $\zeta$ and three values of $\delta(\diamond: \delta / \lambda=0.2,1: \delta / \lambda=0.5, s: \delta / \lambda=0.8)$. In all cases, $r_{0} / \lambda=1$ is chosen. It is observed that the BCE attained for $\delta / \lambda=0.5$ is the lowest, and that it starts with a rapid decrease, whereafter it attains a constant level.
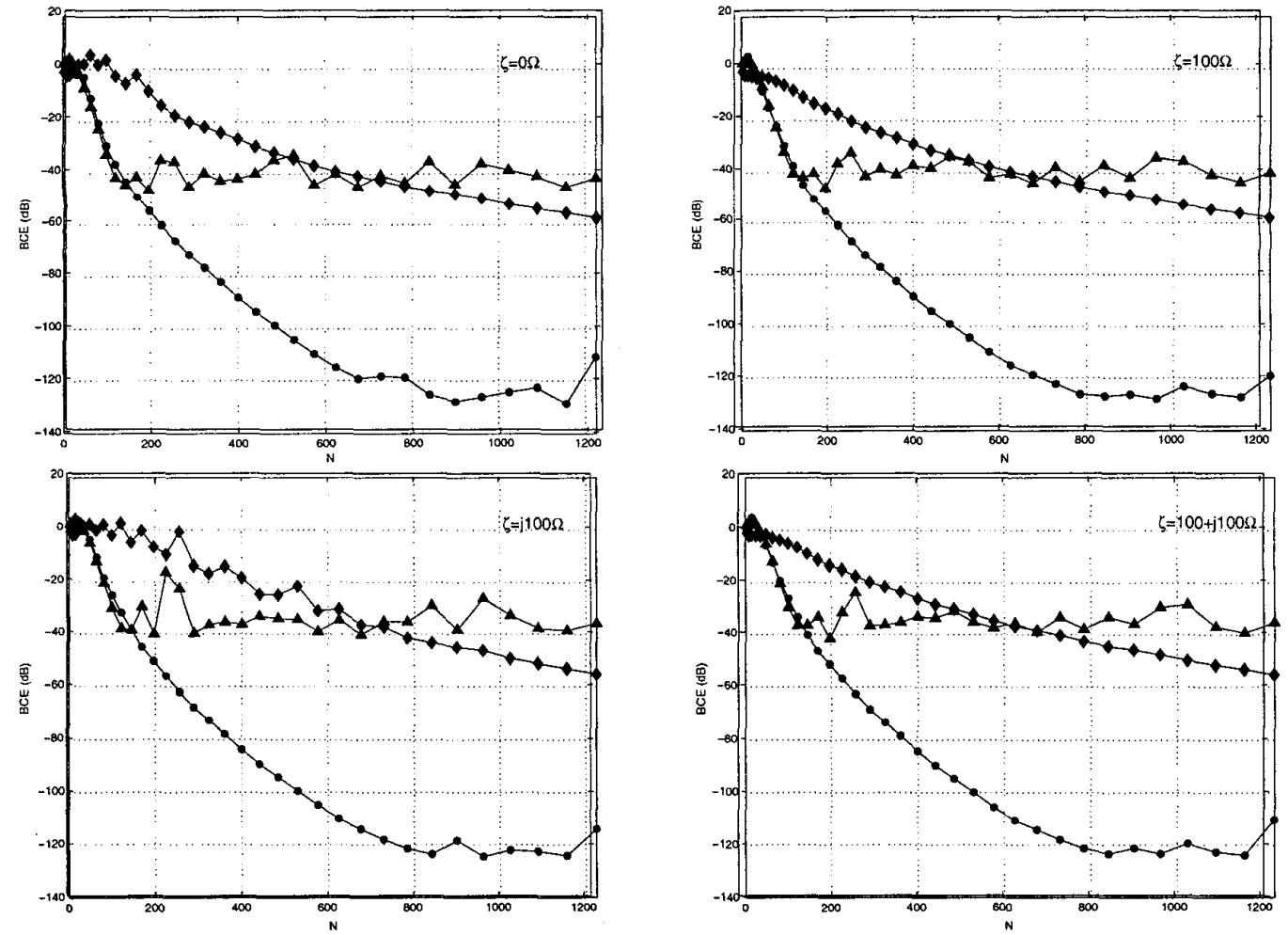

Figure 2 
In Figure 3, the normalised bistatic radar cross section (BRCS) results obtained using MAS for four different scatterers and for two different planes of observation $(\varphi=0$ and $\varphi=\pi / 2)$ are compared with the corresponding SWE results. In all cases, $\zeta=100+j 100 \Omega$ is chosen. There is a very good correspondence between the MAS and SWE results. The small discrepancies can be diminished further if $N$ is increased.
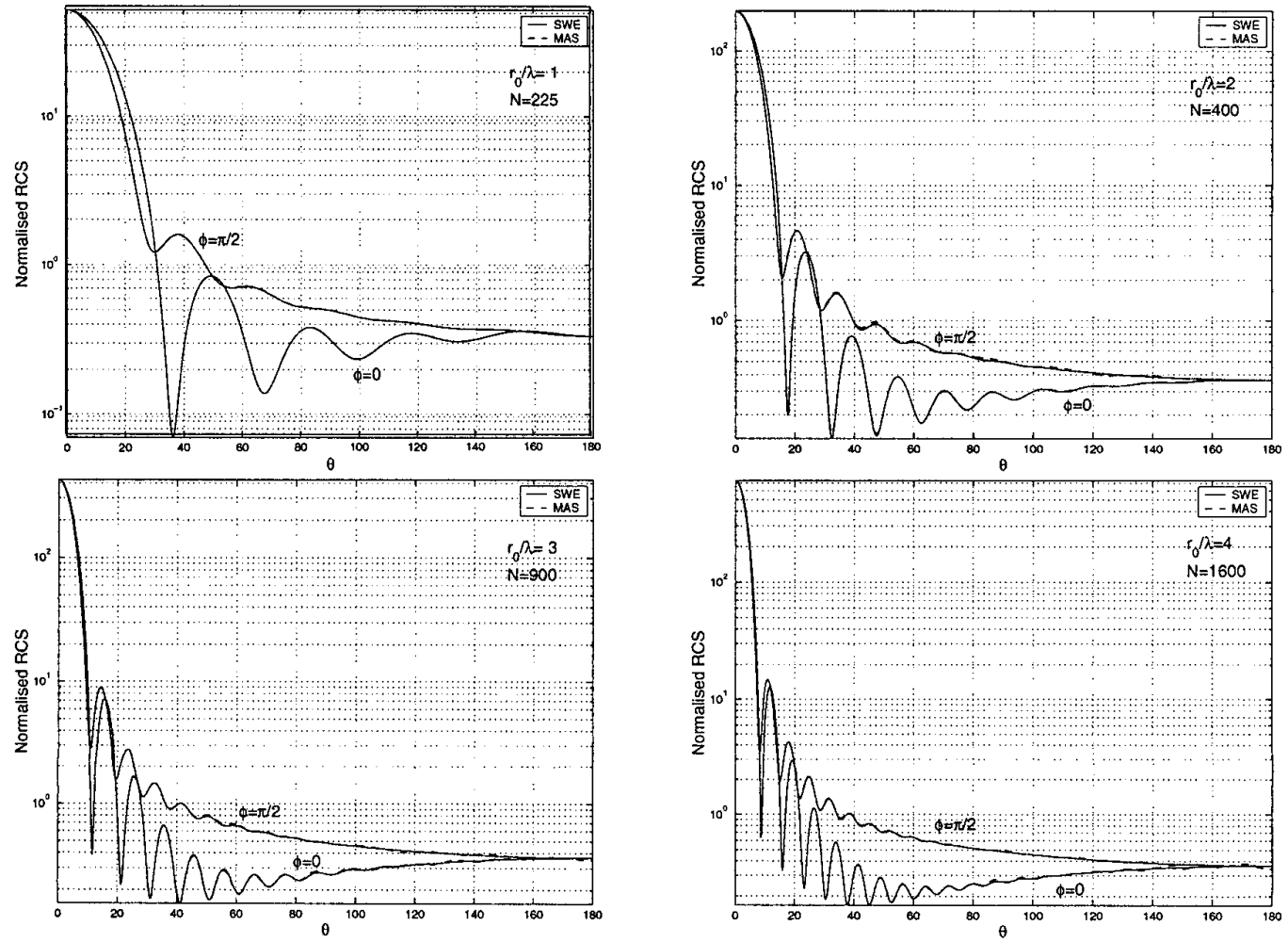

Figure 3

\section{CONCLUSION}

It is seen that the initial decrease in the boundary condition error of the MAS numerical solution is relatively fast. Also, the limiting error level achieved is small enough for the MAS to be able to produce good approximations to exact results at a relatively small computational cost. However, the existence of a finite limit level for the MAS boundary condition error indicates that the present numerical implementation of the method is not convergent. Bistatic radar cross section results obtained by MAS are found to be in good agreement with the reference spherical wave expansion (SWE) solution.

\section{REFERENCES}

[1] Y. Leviatan et al., IEEE Trans. Antennas Propagat., vol. 36, 1722-1734, Dec. 1988

[2] D. I. Kaklamani, European Congress on Computational Methods in Applied Sciences and Engineering, Barcelona 11-14 Sep. 2000

[3] S. Eisler et al., IEE Proceedings, vol. 136, 431-438, Dec. 1989

[4] H. T. Anastassiu et al., IEEE Trans. Antennas Propagat., vol. 50, 59-66, Jan. 2002

[5] Y. Leviatan et al., IEEE Trans. Antennas Propagat., vol. 38, 1259-1263, Aug. 1990

[6] J. E. Hansen, Spherical Near-Field Antenna Measurements, Peter Peregrinus 1988

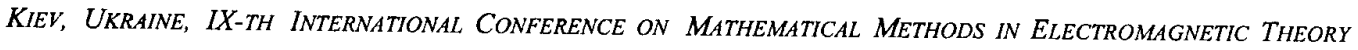

